\title{
Perú y los indicadores económico-sociales y su relación con la marca país ${ }^{1}$
}

DOI: https://doi.org/10.21158/01208160.n89.2020.2820
Pedro Barrientos-Felipa ${ }^{2}$

Universidad Nacional Mayor de San Marcos pbarrientosf@unmsm.edu.pe

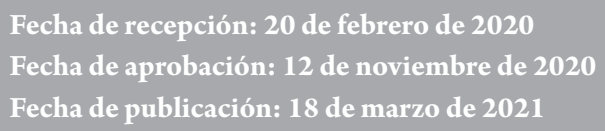

Cómo citar este artículo / To reference this article / Comment citer cet article / Para citar este artigo: Barrientos-Felipa, P. (2020). Perú y los indicadores económico-sociales y su relación con la marca país. Revista Escuela de Administración de Negocios, (89), 91-112. DOI: https://doi.org/10.21158/01208160.n89.2020.2820

\section{Resumen}

La competencia entre países se ha intensificado, motivo por el cual promocionan intensamente la marca país para lograr una mejor posición. A fin de medir su efectividad se consideran indicadores económicos y sociales que permiten conocer los países posicionados, así como aquellos que buscan un lugar preferencial en el comercio internacional. Los indicadores incluyen mediciones del riesgo, la prosperidad y el bienestar de un país, entre otros, que son factores influyentes en el propósito de establecer una marca país. La investigación es de carácter cualitativo, de alcance descriptivo y de diseño no experimental transversal. Se entrevistó con un cuestionario no estructurado a una experta en el tema. Los objetivos del artículo son: a) reconocer la influencia de la marca en la competencia internacional entre países; b) identificar por qué un país debe tener marca y saber administrarla; y c) conocer los factores que permiten generar una marca país. El análisis permite concluir que los países pueden buscar soluciones iniciales si comienzan a preguntarse cómo los países desarrollados, que tienen un posicionamiento internacional, lo lograron; siempre teniendo en cuenta la importancia de emprender cambios estructurales medibles, pues se debe reconocer que este proceso va más allá de la actividad comercial y que tiene como soporte el desarrollo de los sistemas educativo, sanitario y judicial, entre otros, elementos que agregan valor a la imagen del país.

Palabras clave: marca país; comercio internacional; posicionamiento internacional; indicadores de riesgo; indicadores de bienestar; indicadores de prosperidad.

\footnotetext{
Este artículo es consecuencia de la investigación auspiciada por el Vicerrectorado de Investigación y Posgrado de la Universidad Nacional Mayor de San Marcos, «Incidencia de los indicadores económicos y sociales en la formación de la marca país». Se llevó a cabo como parte de las actividades anuales del Grupo de Investigación Ecoagrox, en el periodo 2019. Se presentó en la Reunión Iberoamericana de Socioeconomía-IV RISE: «Transformación productiva, asimetrías territoriales y exclusión social en Iberoamérica». El evento se realizó en la Universidad de Costa Rica, en la ciudad de Heredia.

2 Economista de la Universidad Ricardo Palma, Perú. Magister con Concentración en Marketing de la Universidad del Pacifico, Perú. Doctor en Administración de Negocios Globales en la Universidad Ricardo Palma. Coordinador del Grupo de Investigación Economía y Agroexportación - ECOAGROX. Investigador Rostowrosky - Nivel I CONCYTEC. Miembro del Instituto de Investigaciones Económicas de la Universidad Nacional Mayor de San Marcos, Perú. ORCID: https://orcid.org/0000-0001-52023480
} 


\title{
Peru and economic-social indicators and their relationship with country branding
}

\begin{abstract}
Competition among countries has intensified, making them intensively promote country branding to achieve a better position. In order to measure its effectiveness, we used economic and social indicators to determine the countries that are positioned, and the countries that are seeking a preferential place in international trade. The indicators include measurements of a country's risk, prosperity, and welfare, among others, which are influential factors in the purpose of establishing a country brand. The research is qualitative in nature, descriptive in scope and with a non-experimental cross-sectional design; and includes the interview with an expert on the subject by using an unstructured questionnaire. The aim of this article is: a) to recognize the influence of the brand in international competition among countries; b) to identify why a country should have a brand and to know how to manage it; and c) to know the factors that allow the generation of a country brand. The analysis makes it possible to conclude that countries can seek initial solutions if they ask themselves how developed countries have achieved their international positioning; always bearing in mind the importance of undertaking measurable structural changes, since it must be recognized that this process goes beyond the commercial activity and that it is supported by the development of the educational, health, and judicial systems, among other elements that add value to the country's image.
\end{abstract}

Keywords: country branding; international trade; international positioning; risk indicators; welfare indicators; prosperity indicators.

\section{Peru e os indicadores econômico-sociais e sua relação com a marca-país}

\section{Resumo}

A competição entre os países se intensificou, razão pela qual promovem intensamente a marca-país para alcançar um melhor posicionamento. Para medir a sua eficácia, são considerados indicadores econômicos e sociais que nos permitem conhecer os países posicionados, bem como aqueles que procuram um lugar preferencial no comércio internacional. Os indicadores incluem medidas de risco, prosperidade e o bemestar de um país, entre outros, que são fatores de influência no propósito de estabelecer uma marca-país. A pesquisa é qualitativa, de escopo descritivo e tem um desenho transversal não experimental. Uma especialista no assunto foi entrevistada com um questionário não estruturado. Os objetivos do artigo são: a) reconhecer a influência da marca na competição internacional entre países; b) identificar por que um país deve ter uma marca e saber gerenciá-la; e c) conhecer os fatores que permitem gerar uma marca-país. A análise permite concluir que os países podem buscar soluções iniciais se começarem a se perguntar como os países desenvolvidos, que têm uma posição internacional, conseguiram; tendo sempre presente a importância de realizar mudanças estruturais mensuráveis, visto que se deve reconhecer que este processo vai além da atividade comercial e que tem como suporte o desenvolvimento dos sistemas educacional, sanitário e judiciário, entre outros, elementos que agregam valor à imagem do país.

Palavras-chave: marca-país; comércio internacional; posicionamento internacional; indicadores de risco; indicadores de bem-estar; indicadores de prosperidade. 


\section{Indicateurs socio-économiques et marque pays le cas du Pérou}

\section{Résumé}

La concurrence entre pays, qui s'est fortement intensifiée, oblige à promouvoir fortement les marque-pays pour l'obtention d'une meilleure place au classement des nations. Certains indicateurs économiques et sociaux permettent de connaitre cette place dans les classements internationaux du commerce mondial. Ces indicateurs mesurent principalement les risques, la prospérité et le bien-être de la population d'un pays afin d'établir les marques nationales. Cette investigation est de type qualitatif, descriptif, de conception transversale, non expérimentale. Les objectifs de cet article sont les suivants : a) reconnaître l'influence de la marque-pays dans la compétition internationale des nations ; b) identifier les raisons pour lesquelles un pays devrait investir dans une marque et en assurer la pérennité ; et c) connaître les facteurs permettant la création d'une marque-pays. L'étude conclut que certains pays peuvent trouver des solutions initiales à cette problématique en analysant la façon dont les pays développés, possédant une marque-pays positionnée internationalement, y sont parvenus, et en ayant conscience de l'importance d'entreprendre des changements structurels mesurables. Le développement des systèmes éducatifs, sanitaires et judiciaires valorise ce processus allant au-delà de la simple activité commerciale des pays.

Mots-clés: marque-pays; commerce international; positionnement international; indicateurs de risque; indicateurs de bien-être; indicateurs de prospérité. 
Nation branding is an exciting, complex and controversial phenomenon.

Yvonne Johnston

\section{Introducción : equilibrio del desarrollo de la marca país}

$\mathrm{E}_{\mathrm{n}}^{\mathrm{n}}$ n este artículo se explica cómo establecer una marca país corresponde a un esfuerzo de largo plazo, en el que los decisores de política nacional, policy makers, asumen la realización de cambios estructurales que lo permitan. Establecer la marca país va más allá de campañas publicitarias o ubicar lugares y costumbres que permitan identificarla. La experiencia de los países desarrollados así lo demuestra. La marca país no pretendellegar a tener la capacidad de competir con las grandes potencias económicas, sino tener una identificación propia en el ámbito internacional con productos no tradicionales. Para los fines del caso, lo desarrollamos en consideración a los indicadores que miden la ubicación de Perú en diversos escenarios que miden organizaciones internacionales.

Una consecuencia de la globalización es que el proceso influye para que los países formen bloques económicos que les permitan realizar entre ellos, individual y colectivamente, acuerdos comerciales, así como tratados de libre comercio que les posibiliten intercambiar sus productos a un menor costo. Esto no implica la disminución de las asimetrías que ocurren entre los participantes, pues hay países que tienen una mayor capacidad de negociación frente a otros del mismo bloque, es decir, algunos miembros imponen determinadas reglas de comercio en los otros integrantes.

Este desequilibrio (véase la Figura 1) a favor de algunos países se origina como consecuencia de su sistema de producción asociado a su capacidad de transformación de la materia prima, acceso a capitales, desarrollo de intangibles y la posibilidad de promover nueva tecnología. Estos países tienen una posición ventajosa frente a los demás y, por tanto, en el proceso de negociación, se inicia con ventaja para los países que están mejor posicionados. Con el incremento de la competencia internacional los países deben reflexionar acerca de cómo consideran que los perciben las personas de su propio país y los otros países.

Figura 1. Equilibrio asimétrico entre los integrantes de un bloque económico

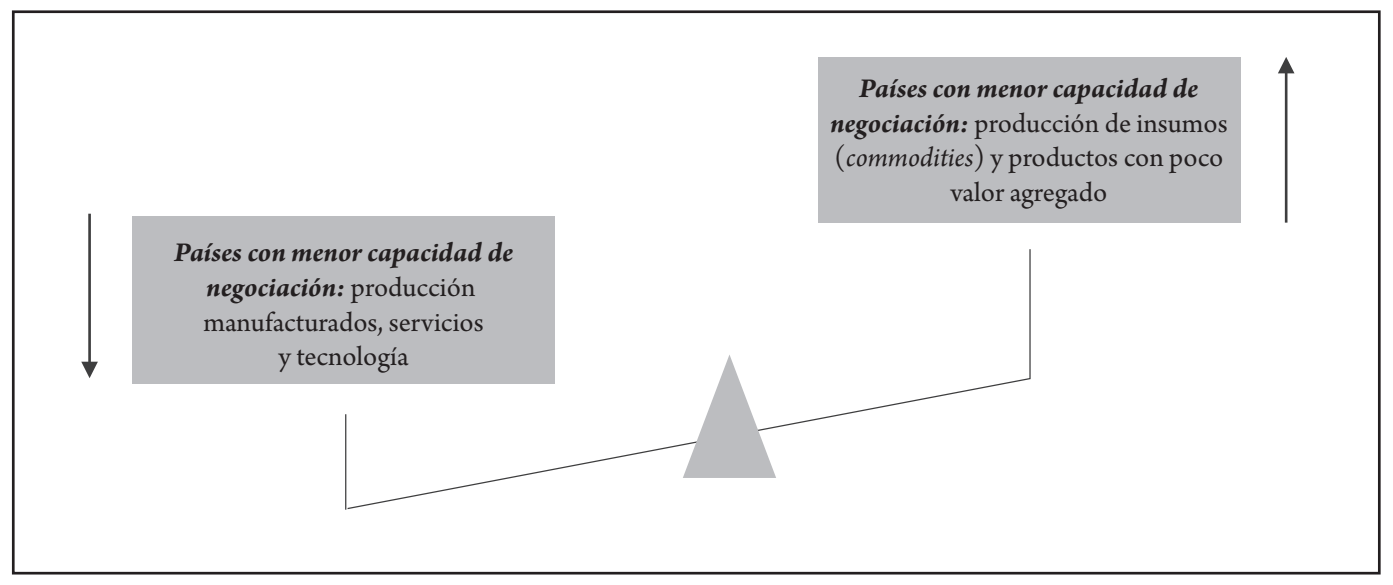

Fuente. Elaboración propia. 
Los países posicionados tienen déficit de insumos en su sistema de producción manufacturera, lo que hace necesario abastecerse de países proveedores, estén o no en el bloque, aunque en los primeros se encuentra la posibilidad del menor costo como resultado de los tratados comerciales. Además de su necesidad de insumos - commodities, materias primas-, para su sistema de producción requieren productos para las familias — productos no tradicionales, con valor agregado o no-.

La demanda actual y potencial origina que los países ofertantes intensifiquen sus acciones de producción y comercio para lograr que el demandante prefiera sus productos al de otros. Así, entonces, la globalización implica que los costos disminuyen, así como los precios, y la competencia aumenta. La formación de bloques no implica que la competencia internacional entre los países integrantes se evita, solo se hace selectiva.

Para el desarrollo y el logro de los objetivos propuestos el artículo expone tres temas. El primero de ellos se refiere a explicar la necesidad de la generación de marca país en consideración a que es un elemento que permite mejores condiciones en la competencia internacional. El segundo tema explica que, además de generar una marca, es importante también saber administrarla. Por último, se detallan los factores que generan la marca país.

\section{Metodología}

Para la realización del presente artículo se optó
por un enfoque cualitativo, en consideración a la naturaleza del tema —-marca país-. Para la obtención de los datos primarios se elaboró un cuestionario no estructurado, y en lo que corresponde a los datos secundarios se identificaron publicaciones relacionadas con la investigación, de organizaciones públicas, instituciones internacionales y artículos de investigadores.

El diseño de la investigación que dio origen al artículo es no experimental transeccional, dado que no se manipularon de forma deliberada las variables y se recolectaron los datos en un momento determinado con la finalidad de describirlas y analizar su influencia en las relaciones de economía internacional. En tal sentido, el alcance de la investigación es de carácter exploratorio descriptivo, para lo cual se aborda la propuesta de Hernández, Fernández y Baptista (2014, p. 90), en cuanto a que «no se deben considerar los alcances como <tipos> de investigación, ya que, más que ser una clasificación, constituyen un continuo de $<$ causalidad > que puede tener un estudio .

En lo que corresponde a los objetivos, se plantean los siguientes: a) reconocer la influencia de la marca en la competencia internacional entre países; b) determinar por qué un país debe tener marca y saber administrarla; y c) conocer los factores que permiten generar una marca país. La elaboración del artículo se basa en el desarrollo - datos secundarios más datos primarios - de los tres objetivos citados. Se añade en el inicio una breve introducción al artículo y se termina con conclusiones acerca del tema.

\section{Competencia internacional y generación de marca}

工entre países para que sus productos se compren en el exterior, como es el caso de Perú, se da en los denominados productos no tradicionales (PNT), en consideración a que los commodities tienen un sistema comercial que se regula a través de las bolsas de valores internacionales. Perú es un importante proveedor de commodities mineros, lo que lo hace atractivo para los inversionistas de este tipo de negocio. 
En promedio, el $70 \%$ de sus exportaciones se relacionan con commodities (véase la Tabla 1) lo cual es un riesgo para el país, pues existe una dependencia en cuanto al precio internacional de esos productos. Por tanto, la intensidad de competencia se da entre países que tienen características similares de producción y comercialización en productos no tradicionales. Además, es a través de este tipo de productos que un país tiene la base para desarrollar una marca.

Tabla 1. Balanza Comercial de Perú — valores FOB en millones de USD_

\begin{tabular}{|c|c|c|c|c|c|c|c|c|}
\hline Años & $\begin{array}{c}\text { Total } \\
\text { exportaciones }\end{array}$ & $\begin{array}{c}\text { Exportaciones } \\
\text { tradicionales-commodities }\end{array}$ & $\begin{array}{c}\text { Exportaciones no } \\
\text { tradicionales }\end{array}$ & Otros & \% A & \% B & $\begin{array}{c}\text { \% } \\
\text { Otros }\end{array}$ & $\begin{array}{c}\text { \% Total } \\
\text { A+B }\end{array}$ \\
\hline 2004 & 11025 & 7942 & 2972 & 111 & 72,04 & 26,96 & 1,01 & 100,00 \\
\hline 2005 & 17368 & 12950 & 4277 & 141 & 74,56 & 24,63 & 0,81 & 100,00 \\
\hline 2006 & 23830 & 18461 & 5279 & 91 & 77,47 & 22,15 & 0,38 & 100,00 \\
\hline 2007 & 28094 & 21666 & 6313 & 114 & 77,12 & 22,47 & 0,41 & 100,00 \\
\hline 2008 & 31018 & 23266 & 7562 & 190 & 75,01 & 24,38 & 0,61 & 100,00 \\
\hline 2009 & 27071 & 20270 & 6196 & 154 & 76,54 & 22,89 & 0,57 & 100,00 \\
\hline 2010 & 35803 & 27850 & 7699 & 254 & 77,79 & 21,50 & 0,71 & 100,00 \\
\hline 2011 & 46376 & 35896 & 10176 & 304 & 77,40 & 21,94 & 0,66 & 100,00 \\
\hline 2012 & 47411 & 35869 & 11197 & 345 & 75,66 & 23,62 & 0,73 & 100,00 \\
\hline 2013 & 42861 & 31553 & 11069 & 238 & 73,62 & 25,83 & 0,56 & 100,00 \\
\hline 2014 & 39533 & 27686 & 11677 & 171 & 70,03 & 29,54 & 0,43 & 100,00 \\
\hline 2015 & 34414 & 23432 & 10895 & 88 & 68,09 & 31,66 & 0,26 & 100,00 \\
\hline 2016 & 37082 & 26183 & 10798 & 101 & 70,61 & 29,12 & 0,27 & 100,00 \\
\hline 2017 & 45422 & 33566 & 11725 & 130 & 73,90 & 25,81 & 0,29 & 100,00 \\
\hline 2018 & 49066 & 35638 & 13240 & 189 & 72,63 & 26,98 & 0,39 & 100,00 \\
\hline
\end{tabular}

A: Exportaciones tradicionales-commodities / B: Exportaciones no tradicionales

Fuente. Elaboración propia con base en las Memorias Institucionales del Banco Central de Reserva del Perú.

En este entorno, para los países es conveniente poseer capacidad de diferenciar su oferta frente a otros competidores como una actividad sostenible y permanente. Los países con una industria manufacturera consolidada internacionalmente ya tienen esa ventaja, la cual han generado mediante un proceso de actividades comerciales a lo largo del tiempo, incluso con aplicación de acciones proteccionistas orientadas a permitir el crecimiento de las nuevas actividades industriales en su propio territorio. Son países que ya tienen un claro posicionamiento.

En cambio, en el caso de los países exportadores de insumos, el posicionamiento lo tienen a un nivel básico, pues se caracterizan por tener su actividad productiva relacionada con productos con poco o sin valor agregado, lo que influye en el crecimiento económico y en los negocios internacionales. Para ambos casos, países industrializados y países productores de insumos, Kotler y Keller (2012) denominan a este tipo de posicionamiento como bidireccional y se puede relacionar a que el país tiene dos puntos de referencia: commodities y productos no tradicionales. La figura 2 expone la bidireccionalidad de un país que se caracteriza por producción de insumos, en el que el punto de referencia indica la tendencia de la producción exportable. 
Figura 2. Bidireccionalidad de la producción exportable

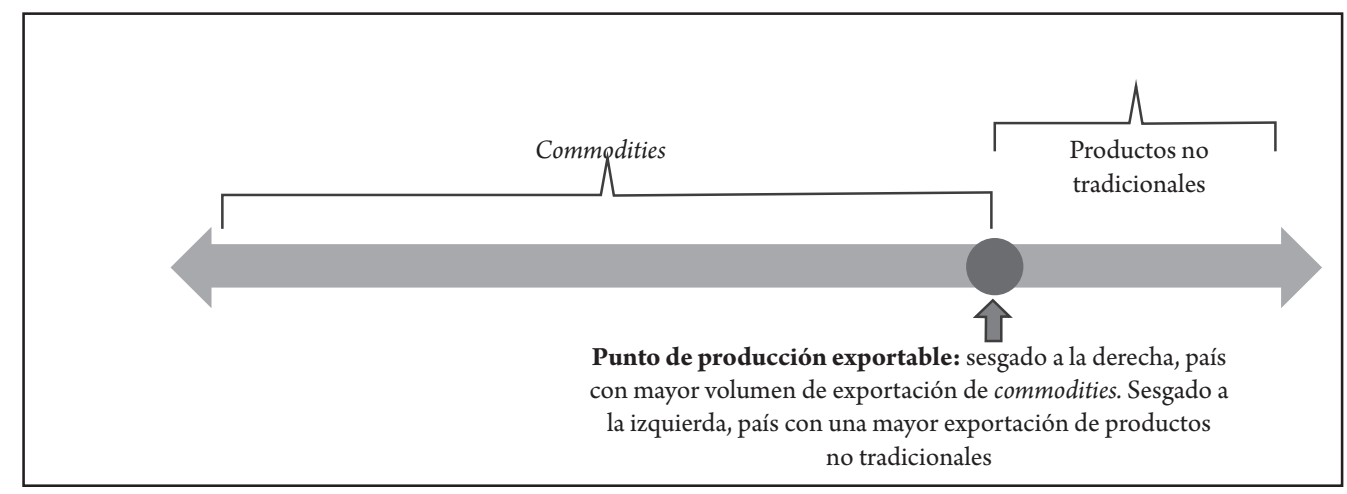

Fuente. Elaboración propia.

La posición bidireccional es una oportunidad para que los países modifiquen porcentualmente su matriz de producción, de modo que disminuya su riesgo ante los cambios bruscos de precios internacionales, en especial cuando el país tiene una mayor influencia en la exportación de commodities y también hace posible una mayor cobertura del comercio internacional. Sin embargo, si no se hacen esfuerzos significativos, la posición con mayor valor económico determina la posición que el país tiene para los compradores e inversionistas. Por tanto, Perú puede asumir que tiene una mayor posición relacionada con un productor de commodities, un país que tiene poca manufactura o de poco valor agregado en sus productos de exportación y, por tanto, mayor riesgo a cambios bruscos en el comportamiento de los compradores.

La habilidad de los hacedores de la política económica debe tener como consecuencia que la bidireccionalidad se modifica a través de cambios en el patrón de producción del país, en procura de una mayor ascendencia relativa o porcentual de productos manufacturados o con mayor valor agregado tanto en el campo de los bienes como en los servicios para el mercado internacional. Los países exitosos se destacan por esa estrategia, pues diseñan e implementan políticas, actividades y programas que tienen como objetivo maximizar el valor que sus ciudadanos perciben de su país de origen, así como de aquellos con los cuales realiza actividades comerciales. La figura 2 también expone la decisión que deben tomar los hacedores de política en cuanto a si movilizan el «circulo» hacia la izquierda o continúan en la situación actual.

Es así como los países inician intencionalmente la creación de una marca país, en un proceso que demora periodos extensos de tiempo y aplica la promoción -que incluye la publicidad- o comunicación planificada como una actividad que apoya la generación de esta. La generación de una marca país necesita de un mayor esfuerzo interno que le permita generarla a través de sus PNT. La publicidad no genera marca, la hace conocida. La marca la genera el esfuerzo común del país en modificar el patrón de producción. Queda la posibilidad de preguntar si es lo que el país necesita para que sus ciudadanos tengan un mejor bienestar a través de las exportaciones de productos manufacturados o con valor agregado, lo cual es considerar el camino que los denominados países desarrollados realizaron.

Elesfuerzo que realiza un país implica tomar decisiones acerca de qué posición considera lograr dentro del proceso de globalización. Lo que el país realiza o deja de hacer en el corto plazo comunica sus intenciones de largo plazo. Si fuera así, entonces actúa con base en la inmediatez, se actúa coyunturalmente o solo para el momento, sin un objetivo definido, lo que en el largo plazo afecta sus objetivos de un posicionamiento que otorgue valor al país y, por tanto, a sus ciudadanos. 
De ahí la complejidad de establecer una marca país. Tener marca país en el sistema de comercio global significa que se tiene en la comunidad internacional un posicionamiento claro, con ventaja frente a otros países y empresas. Los compradores e inversionistas locales y extranjeros constituyen la principal fuente para generar los ingresos que el país necesita para llevar a cabo sus actividades de creación de valor en beneficio de sus ciudadanos.

Los países exitosos invierten en sus ciudadanos, quienes son el soporte de cualquier actividad económica que se realice. De hecho, están en el primer nivel de importancia si se considera que es a través de ellos que se atrae compradores e inversionistas, pues son sus capacidades lo que permite atraerlos, en especial cuando se trata de productos que requieren valor agregado, de modo que representan la identidad del país. Por tanto, la principal actividad que cumple un Estado es satisfacer las necesidades de sus ciudadanos, para lo cual diseña un proceso continuo de beneficios y asunción de costos para que se identifiquen con las acciones que se realizan en el contexto del comercio internacional.

La figura 3 expone la prioridad en la formación de la marca país, de manera que identifica a los ciudadanos en la base de toda acción. Menciona Porter (2009, p. 221) que la «competitividad de una nación depende de la capacidad de su industria para innovar y mejorar». Para este caso se le debe dotar de recursos que lo permitan, de ahí la importancia de la necesidad de fortalecer el bienestar de los ciudadanos, la cual puede «tangibilizarse» a través de indicadores socioeconómicos desarrollados por instituciones representativas. Internamente, también es importante fortalecer la capacidad del empresariado nacional de acuerdo con las posibilidades y las necesidades del país. La figura 3 expresa que la prioridad en un país está en sus ciudadanos y sus empresas, y través de estas últimas relacionarse con el mercado de bienes, servicios, inversión y financiero.

Figura 3. Prioridades en la formación de marca país

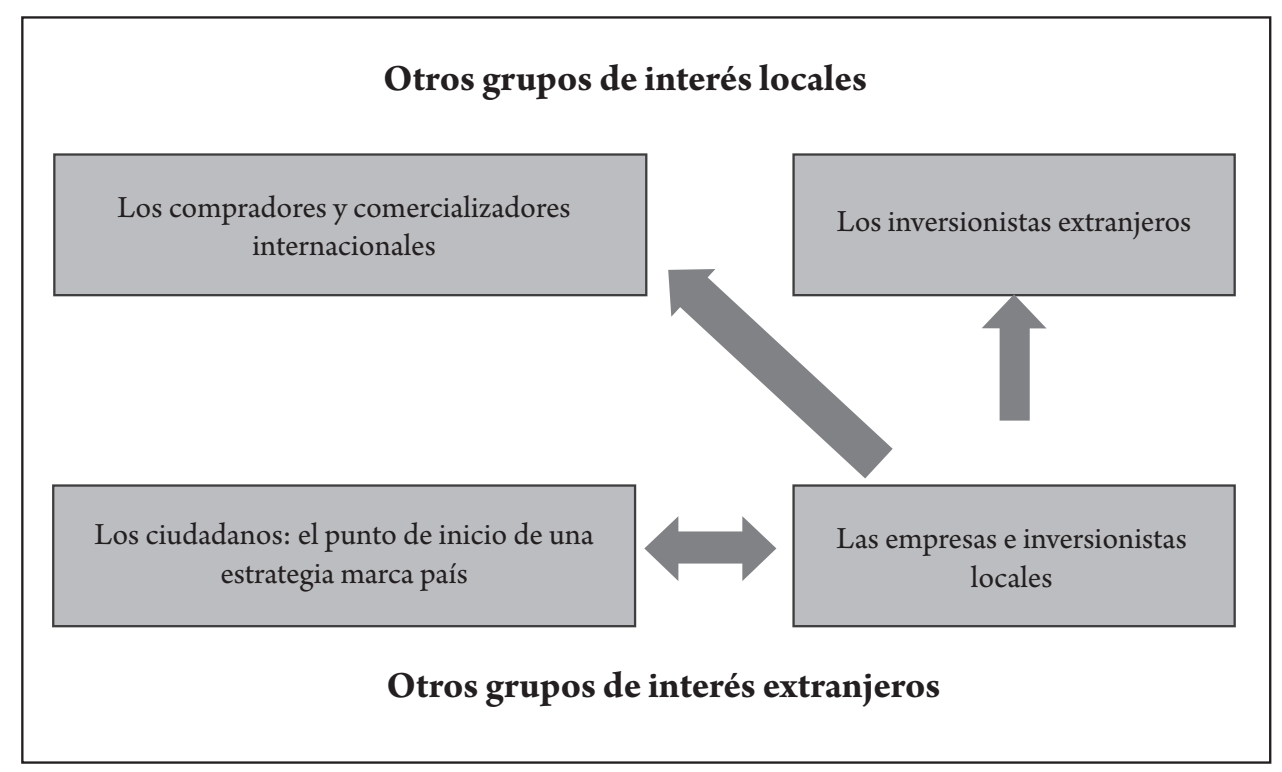

Fuente. Elaboración propia.

Ohmae (2005, p. 281) explica que «un gobierno verdaderamente visionario se dedica a la educación». Esto implica que el gobierno debe comprometerse más en la educación, en todos sus niveles —básico, secundario, universitario o técnico-, de modo que quedé por decidir por qué nivel educativo se inicia, considerando la limitación de presupuesto, los objetivos de los grupos de referencia y la capacidad de 
los instructores. Es un cambio estructural necesario con miras a que se establezca una marca país, pues en el caso contrario todo esfuerzo por establecerla queda en una campaña publicitaria agradable. También menciona que «la educación tiene beneficios tangibles: una fuerza de trabajo altamente educada es una parte necesaria de cualquier economía, pues añade valor intelectual a lo que produzca o proporcione».

Cuando se cita el concepto competir se hace alusión a hacerlo con otros países de similares características económicas y sociales, los cuales, al comportarse como ofertantes buscan obtener la accesibilidad a los compradores actuales para que aumenten su demanda; así mismo, a nuevos mercados, mediante el diseño de estrategias que hagan atractivos sus productos, incluso con diseños a medida. La competencia internacional de productos no ocurre a través de commodities, sino a través de los productos no tradicionales.

Aunque la competencia aumenta relativamente los costos, si se le enfrenta de manera estratégica genera el triple beneficio: financiero, de aprendizaje y de valor. Financiero porque al lograr imponerse al competidor con una mejor oferta al comprador se genera una ganancia; de aprendizaje, porque la empresa aprende cómo hacer mejor las cosas con miras a administrar de forma eficiente la productividad; de valor, porque es el que genera satisfacción al cliente y es el que asegura el posicionamiento internacional del país.

La globalización influye en los países para que estén interconectados, de manera que permite realizar transacciones comerciales más fluidas. Más aún, con la evolución de la tecnología, en la que los tiempos de comunicación y transacción disminuyen las barreras comerciales de una forma sorprendente, los países están presionados a mejorar sus ofertas. $\mathrm{Si}$ se considera que el incremento de transacciones aumenta, el país debe tener conocimiento de cuánto favorece a sus ciudadanos la mejora de servicios que el Estado les otorga. El comercio internacional favorece a los productores, comercializadores, inversionistas y ciudadanos como punto de origen, así como al Estado que apoya el comercio internacional. Cuando se inician las actividades orientadas a establecer una marca país, los diseñadores saben que debe realizarse la evaluación de los resultados y la consecuencia en los grupos mencionados. El de un país es un proceso de continua evaluación de resultados.

El establecimiento de la marca país es una actividad de marketing país, por tanto, deben evaluarse las estrategias y las tácticas considerando tal situación. $\mathrm{Al}$ respecto debe tenerse en cuenta el alcance de este concepto, el cual Buitrago (2010, p. 28) define como el «conjunto de percepciones que caracterizan de manera instantánea a un país, es como una carta de presentación natural, que de acuerdo a diferentes posiciones puede desempeñar un papel a favor o en contra». Además, menciona que el resultado genera un «paraguas $\gg$ que favorece al empresariado local, los productos - bienes y servicios-, a sus ciudadanos, así como las interrelaciones públicas y privadas a nivel internacional.

Un indicador que sirve para la evaluación macro, con respecto a los ciudadanos, es el Índice de Desarrollo Humano (IDH), el cual expone qué tanto beneficio reciben los ciudadanos para mejorar sus condiciones de vida. El IDH es un conjunto de índices e indicadores presentados en un informe del Programa de las Naciones Unidas para el Desarrollo (PNUD) (2018, p. 1). De acuerdo con este programa, «el desarrollo humano tiene que ver con las libertades humanas. Consiste en desarrollar las capacidades humanas, no solo de unos pocos, ni siquiera de la mayoría, sino de todas las personas».

Este último informe considera 189 países (p. 2), de los cuales $\ll 59$ pertenecen al grupo de desarrollo humano muy alto, 53 al de desarrollo humano alto, 39 al de desarrollo humano medio y solo 38 al de desarrollo humano bajo». Tomar en cuenta este indicador es tener conocimiento de las posibilidades que tienen los ciudadanos y las oportunidades que tienen los hacedores de políticas para mejorar los resultados y dar sentido a la creación de una identidad nacional y de una marca país. El documento expone lo siguiente: 
El Índice de Desarrollo Humano (IDH) es un índice compuesto que se centra en tres dimensiones básicas del desarrollo humano: la capacidad de tener una vida larga y saludable, que se mide por la esperanza de vida al nacer; la capacidad de adquirir conocimientos, que se mide por los años promedio de escolaridad y los años esperados de escolaridad; y la capacidad de lograr un nivel de vida digno, que se mide por el ingreso nacional bruto per cápita. $(2018$, p. 1$)$

La tabla 2 identifica la ubicación que le corresponde a los países latinoamericanos en esta evaluación. Los tres países mejor ubicados son Chile -44-, Argentina -47- y Uruguay -55-, los cuales se encuentran en la categoría «Muy alto»; Perú — 89 — está en la categoría «Alto». Los resultados son una muestra de la oportunidad que tienen los ciudadanos de un país de apoyar la generación de una marca, cuya contrapartida son los beneficios que recibe este en cuanto a mejora de ingresos reales, salud y educación, que son los indicadores que se utilizan para considerar el desarrollo humano. La oportunidad la crean los hacedores de política, es decir, en el interior del país y no desde el exterior. Si la intención es crear una marca país consolidada y sostenible, entonces diseñan y ponen en práctica las acciones que son necesarias. Un indicador complementario que puede aplicarse es el Índice de Prosperidad elaborado por el Instituto Legatum, quienes expresan (2019, p, 2):

Our mission at the Legatum Institute is to create the pathways from poverty to prosperity, by focussing on understanding how prosperity is created and perpetuated. Prosperity entails much more than wealth: it reaches beyond the financial into the political, the judicial, and the wellbeing and character of a nation -it is about creating an environment where a person is able to reach their full potential-.

En este índice, Perú, en el 2009, ocupa el puesto 62, en el 2018 el lugar 57 y en el 2019 el 56. Los indicadores que toma en cuenta son la seguridad, la libertad personal, la gobernanza, el capital social, el ambiente para invertir, las condiciones empresariales, el acceso al mercado y la infraestructura, la calidad de la economía, las condiciones de vida, la salud, la educación y el ambiente natural.

Tabla 2. Ubicación de Perú en el Índice de Desarrollo Humano

\begin{tabular}{|c|c|c|c|c|c|c|c|c|}
\hline $\mathbf{N}^{\circ}$ & País & $\begin{array}{l}\text { IDH } \\
2017\end{array}$ & $\begin{array}{l}\text { EVN } \\
2017\end{array}$ & $\begin{array}{l}\text { AEE } \\
2017\end{array}$ & $\begin{array}{l}\text { APE } \\
2017\end{array}$ & $\begin{array}{l}\text { INB en USD de } \\
2011-2017\end{array}$ & $\begin{array}{c}\text { Clasificación } \\
\text { según el INB } 2017\end{array}$ & $\begin{array}{c}\text { Clasificación según } \\
\text { el IDH } 2016\end{array}$ \\
\hline 44 & Chile & 0,843 & 79,7 & 16,4 & 10,3 & 21,910 & 13 & 44 \\
\hline 47 & Argentina & 0,825 & 76,7 & 17,4 & 9,9 & 18,461 & 19 & 47 \\
\hline 55 & Uruguay & 0,804 & 77,6 & 15,9 & 8,7 & 19,930 & 5 & 56 \\
\hline 63 & Costa Rica & 0,794 & 80,0 & 15,4 & 8,8 & 14,636 & 15 & 63 \\
\hline 66 & Panamá & 0,789 & 78,2 & 12,7 & 10,2 & 19,178 & -4 & 66 \\
\hline 73 & Cuba & 0,777 & 79,9 & 14,0 & 11,8 & 7,524 & 43 & 72 \\
\hline 74 & México & 0,774 & 77,3 & 14,1 & 8,6 & 16,944 & -6 & 74 \\
\hline 78 & Venezuela & 0,761 & 74,7 & 14,3 & 10,3 & 10,672 & 20 & 77 \\
\hline 79 & Brasil & 0,759 & 75,7 & 15,4 & 7,8 & 13,756 & 2 & 79 \\
\hline 86 & Ecuador & 0,753 & 76,6 & 14,7 & 8,7 & 10,347 & 15 & 84 \\
\hline
\end{tabular}




\begin{tabular}{|c|c|c|c|c|c|c|c|c|}
\hline 89 & Perú & 0,750 & 75,2 & 13,8 & 9,2 & 11,789 & 3 & 86 \\
\hline 90 & Colombia & 0,747 & 74,6 & 14,4 & 8,3 & 12,938 & 4 & 89 \\
\hline 94 & $\begin{array}{c}\text { República } \\
\text { Dominicana }\end{array}$ & 0,736 & 74,0 & 13,7 & 7,8 & 13,921 & -15 & 95 \\
\hline 110 & Paraguay & 0,702 & 73,2 & 12,7 & 8,4 & 8,380 & -2 & 108 \\
\hline
\end{tabular}

Nota: Chile, Argentina y Uruguay, de acuerdo con el Índice de Desarrollo Humano, se ubican en la categoría «Muy alto». Los once siguientes países latinoamericanos se ubican en la categoría «Alto».

IDH: Índice de Desarrollo Humano / EVN: Esperanza de vida al nacer (Años) / AEE: Años esperados de escolaridad / APR: Años promedio de escolaridad / INB: Ingreso nacional bruto per cápita en USD de 2011 - 2017 / Clasificación según INB: Clasificación según el INB per cápita menos la clasificación en función del IDH

Fuente. Elaboración propia con base en datos del Programa de las Naciones Unidas para el Desarrollo, PNUD, 2018.

El IDH es un indicador de la situación de los ciudadanos en su país, de cómo es que a ellos los atiende el Estado. En los países que son marca, es decir, aquellos que tienen un posicionamiento consolidado, para sus compradores o vendedores los estándares de salud, educación y de ingreso no representan problema, pues sus ciudadanos se satisfacen de forma adecuada con los servicios relacionados con salud, escolaridad y trabajo, que son tareas que el Estado debe cumplir. Al tomar en cuenta los estándares de Australia -83,1; 12,9; 43,560 - y compararlos con Perú — 75,2; 9,2; 11,789- podemos concluir el esfuerzo realizado por los gobiernos respectivos. Los resultados son consecuencia del accionar a largo plazo, en los que el país, expresado a través de sus fuerzas dirigentes o los tomadores de decisión política y económica, tienen conocimiento de qué lugar deben ocupar en el contexto internacional.

En el establecimiento de la marca país también es importante conocer cómo perciben a un país los inversionistas, quienes son precavidos acerca de sus decisiones y hacia dónde dirigen sus capitales, por tanto, miden el riesgo que tiene un país para sus inversiones mediante diversos conceptos cuantitativos. En opinión de Iranzo (2008, p, 12), el concepto riesgo país se relaciona con situaciones acerca de lo que ocurre en un país determinado en su economía, sus finanzas, su política, su historia y su sociología. El concepto que aplica la autora es: «El riesgo país es la probabilidad de que se produzca una pérdida financiera por circunstancias macroeconómicas, políticas o sociales, o por desastres naturales, en un país determinado». Para el cálculo de un indicador, el Ministerio de Economía y Finanzas de Perú (2002, p, 35) señala:

podemos hallar una medida de riesgo país o prima por riesgo país a través de un diferencial de rendimientos al vencimiento - <yield to maturity $>$ - entre un bono soberano denominado en dólares emitido por un país emergente y un bono de los Estados Unidos de vencimiento equivalente.

Barrientos (2014) explica que existe una diversidad de modelos para medir el riesgo país, al poner en consideración los conceptos - en muchos casos son similares - que se aplican para llegar a determinar un índice, el cual determina un ranquin del riesgo que asumen los inversionistas internacionales para canalizar su capital a determinado país, en una determinada actividad.

Estos conceptos de evaluación representan, para el establecimiento de la marca, los factores de diferenciación que, al ser evaluados, se ubican en un espectro cuantitativo de lo favorable y desfavorable; si es favorable se invierte, en el caso contrario se da el alejamiento de los inversionistas. Los países, a través de la política económica, procuran obtener resultados favorables de acuerdo con estos índices y así considerar 
la atracción oportuna de inversión privada, incluso de apoyo financiero de otros gobiernos e instituciones financieras.

En la figura 4 se observan diez conceptos que permiten evaluar el riesgo de invertir en un país. Así, si el sesgo es hacia la derecha, las posibilidades de inversión local y extranjera son pocas, mientras si es más la tendencia al extremo derecho la posibilidad de inversión privada es menor, a no ser que se considere oportuno asumir un alto riesgo. En el caso contrario, si el riesgo tiene una tendencia a la izquierda del gráfico las posibilidades de que se canalicen recursos hacia estos países son mayores. Los que tienen resultados positivos son los países que tienen un posicionamiento que estimula el flujo de inversiones, con lo que se propicia así el crecimiento del país; si los resultados van hacia el extremo izquierdo el riesgo disminuye.

Este es uno de los beneficios cuando un país se convierte en marca, toda vez que permite a los decisores tomar una rápida elección frente a las propuestas que se le presentan de diversos países y organizaciones a nivel mundial. La marca se convierte en una referencia de buena actuación interna y externa del país, en un ambiente en el que cada vez la competencia se intensifica. Al respecto, Labarca (comunicación personal, julio de 2019) opina que, a pesar de una intensa competencia por atraer capitales, «si hay diferenciación y estrategia, hay espacio para todos».

Figura 4. Conceptos para medir el riesgo país

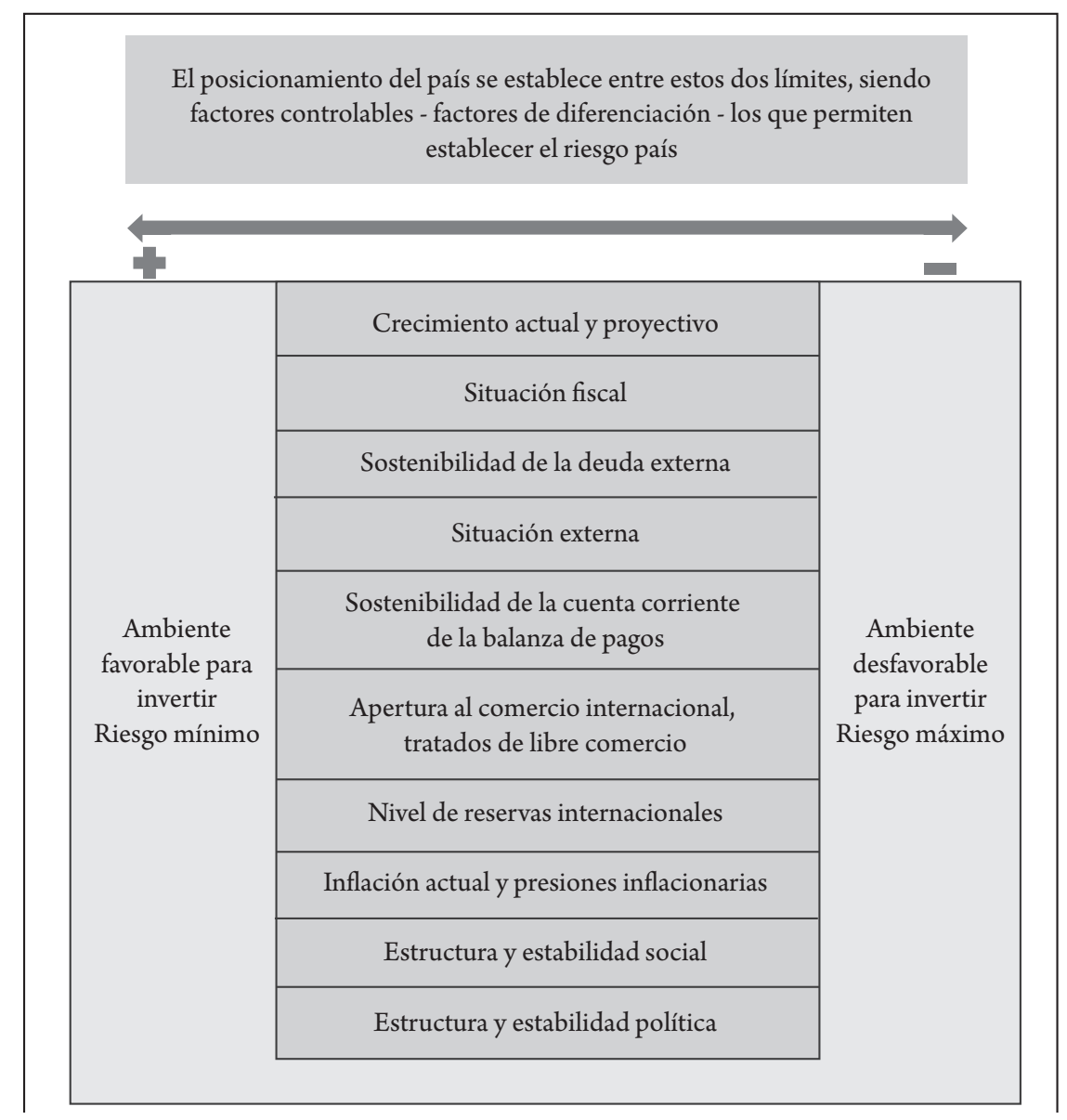

Fuente. Elaboración propia con base en Barrientos, 2014, p. 123. 
Se puede considerar que la realización de una marca país es un proceso complejo que implica, en su parte inicial, el reconocimiento de una serie de carencias que son parte del país en el momento en que decide establecerse - a través de las fuerzas que le dan vida - como un país que puede ser distinto y mejor que otros de similar nivel socioeconómico. Establecer una marca país no significa considerar que el país logre los estándares de los países desarrollados, sino que es un país diferenciado, que soluciona sus problemas con las mejores alternativas posibles.

Kotler, Jatuspitak y Maesincee (1998) citan a Hieronymi ${ }^{3}$, quien realiza un análisis de la política económica en Hungría, y mencionan la circunstancia previa a la decisión que ocurre en un país cuando quiere hacer mejor las cosas o establecerse como una marca país. Esto es, el momento en que los dirigentes del país tienen una visión coherente de lo que debe ser el país y asumen un comportamiento optimista de las contradicciones que puede haber entre ellos. Los países parten del posicionamiento que tienen en la comunidad internacional, lo que habitualmente también se relaciona con lo expuesto en la figura 4.

Al lograr resultados satisfactorios, económicos y sociales, los países tienen la posibilidad de buscar establecerse con una imagen país, pues tal acción los vuelve atractivos a una diversidad de agentes internacionales. Así lo señala Fetscherin (2010), quien también opina que esta es una de las razones de estos tiempos en los que los países hacen uso del marketing para lograr ventajas, asumiendo que este proceso es complejo, excitante y controversial. En el extremo, toda acción de un país - política, económica social- tiene efecto en su imagen internacional. También señala que la literatura del marketing puede entenderse por medio de cuatro conceptos: country of origin, destination branding, country image or country-product image y country identity.

La idea central de Fetscherin (2010) puede resumirse en considerar que el mundo es cada vez más complejo y sus integrantes están en una continua búsqueda de interrelacionarse, aun considerando que entre ellos compiten en todo nivel. Cita a Anholt (p. 234), quien manifiesta que «la globalización está convirtiendo al mundo en un supermercado inmenso», de modo que se da la intensificación de la competencia y acciones de los países por diferenciarse y así establecer una ventaja competitiva con la seguridad de que lograr una marca país les permite contribuir a detener un desarrollo sostenible.

\section{Un país debe tener marca y saber administrarla}

Ci se considera lo propuesto por Fetscherin $\mathcal{O}(2010)$, quien a su vez toma las ideas de Anholt (2004), en cuanto a cómo los países que compiten internacionalmente actúan como si fueran gigantescos supermercados, entonces, bajo tal premisa puede considerarse que los países deben alinear su oferta a través de sus diversas líneas de negocios — sectores económicos-, sus sublíneas — sectores industriales - y los diversos productos —bienes, servicios, lugares, personalidades — que se encuentran en cada uno de ellos. Sin embargo, se debe considerar la opinión de Kaneva (2011) en cuanto a que la marca país no implica acciones de propaganda ni acciones mediáticas para influir en la opinión pública. Su opinión implica que desarrollar una marca es un proceso serio y estratégico, en el que la publicidad y las relaciones públicas son instrumentos de apoyo y no la finalidad.

\footnotetext{
3 Licenciado en Economía Internacional. Profesor Investigador de Webster University en el tema de relaciones internacionales. Tiene numerosas publicaciones — libros, artículos y reportes de investigación-.
} 
La creación de una marca país es un proceso de largo aliento y la publicidad es una parte de este. Un país, en el propósito de establecerse como marca - lo que implica que la comunidad internacional confía en el país como un proveedor y como comprador- debe asumir determinados retos sociales que le permiten posicionarse con ventajas frente a sus competidores. Indicadores económicos tales como el producto bruto interno, la balanza de pagos, la inflación y la política cambiaria exponen el crecimiento económico del país. Los países pueden tener buenos resultados económicos, pero no se establecen como marca.

Existen otros factores que los hacedores de la política nacional interesados en establecer una marca país deben incluir: los factores que se relacionan con el bienestar de todos los ciudadanos. Los países que tienen marca son aquellos que para la comunidad internacional tienen una identidad que les favorece con base en factores sociales que se relacionan con el bienestar de su comunidad. Labarca (comunicación personal, julio de 2019) expresa que la marca país es «muy importante, sobre todo en aquellos casos en que las exportaciones, la inversión extranjera o el turismo sean esenciales para la economía y el desarrollo económico del país».

Los países que buscan posicionarse o establecerse como una marca realizan actividades que los llevan a intensificar la promoción de sus productos — lugares y costumbres, entre otros- a través de intensas campañas publicitarias tanto en el mismo país como en el extranjero. Existe tal competencia que, incluso, se encuentran estadísticas que exponen quién avanza y quién no, así como también empresas que asesoran en relación a cómo un país puede hacer un mejor marketing y ganar en la competencia. Al promover su oferta los países optan por crear una marca país, para lo cual realizan intensas campañas promocionales con el fin de darla a conocer.

Se puede poner como ejemplo la promoción del turismo que, en términos de marketing, es una línea de negocios compuesta por sublíneas. Dentro de estas se encuentran las situaciones, los lugares y las ciudades específicas de cada país. Sin embargo, este tipo de acción va en sentido contrario a lo que proponen Fetscherin (2010) y Kaneva (2011) en lo que corresponde a formar una imagen país, ya que la limitan a la publicidad, las relaciones y otras variables del marketing, lo cual implica un comportamiento estratégico limitado.

En cuanto a la intensificación de las campañas publicitarias para promover un país determinado, se observa cómo se relacionan más con el turismo o el incremento de visitantes extranjeros, lo que en el interior de un país puede ser un riesgo si se considera que los pobladores pueden asumir que es suficiente para crear una marca país; incluso se crean logotipos como un aval a tal acción.

La marca país es un concepto de marketing cuya construcción es un proceso complejo que hace uso de la publicidad para hacer conocer lo que es el país, lo que se está haciendo y lo que se espera realizar. Se tiene el caso de Bloom Consulting (2018), empresa experta en el desarrollo de marca país, la cual expone acerca de los países que están consolidados en el campo de turismo (véase la Tabla 3) resultados que, al confrontarse con el IDH, muestran que varios de los países que se incluyen son los que tienen un mejor índice. A Perú le corresponde el lugar 41 del ranquin mundial con un $\mathrm{CBS}^{4}$ Rating de A.

4 Sigla para Country Brand Strategy. 
Tabla 3. The global top 25 performers rank

\begin{tabular}{|c|c|c|c|}
\hline $\mathbf{N} .^{\circ}$ & Variación & País & CBS Rating \\
\hline 1 & - & Estados Unidos de América & AA \\
\hline 2 & +4 & Tailandia & A \\
\hline 3 & -1 & España & $\mathrm{BBB}$ \\
\hline 4 & - & Hong Kong & AA \\
\hline 5 & +2 & Australia & A \\
\hline 6 & -1 & Francia & A \\
\hline 7 & +2 & China & AA \\
\hline 8 & -5 & Alemania & AA \\
\hline 9 & +2 & Reino Unido & $\mathrm{BBB}$ \\
\hline 10 & -5 & Italia & $\mathrm{BBB}$ \\
\hline 11 & +2 & Turquía & $\mathrm{BBB}$ \\
\hline 12 & -4 & Macao & AA \\
\hline 13 & - & Singapur & AA \\
\hline 14 & - & Japón & A \\
\hline 15 & +2 & Canadá & A \\
\hline 16 & +4 & México & $\mathrm{BBB}$ \\
\hline 17 & -2 & Suiza & A \\
\hline 18 & -6 & Austria & $\mathrm{AA}$ \\
\hline 19 & +6 & Grecia & A \\
\hline 20 & +2 & Portugal & A \\
\hline 21 & -3 & Corea del Sur & $\mathrm{BBB}$ \\
\hline 22 & +1 & India & $\mathrm{BBB}$ \\
\hline 23 & -4 & Malaysia & $\mathrm{BBB}$ \\
\hline 24 & -3 & Países Bajos & AAA \\
\hline 25 & +1 & Taiwán & A \\
\hline
\end{tabular}

AAA Very strong / AA Strong / A Slightly Strong / BBB Very good / BB Good / B Slightly good / CCC Slightly Weak / CC Weak / C Veryweak / D Poor

Fuente. Elaboración propia con base en Bloom Consulting, 2018.

Otro indicador que puede guiar acerca de las acciones que deben tomarse para la construcción de una marca país es el que se relaciona a la igualdad de ingreso, el cual se basa en la aplicación del coeficiente de Gini. La aplicación de este brinda un retrato acerca de cómo está distribuida la riqueza en un país, sin que sea relevante lo que la caracteriza como sociedad; el resultado de su aplicación muestra que tan equitativamente está distribuida la riqueza en un país. Cuando el resultado es 0 , implica que los ingresos y el consumo se distribuyen de manera equitativa entre los pobladores, cuando tiene una tendencia hacia 1 es todo lo contrario. En la tabla 4 se identifican los primeros veinte países ubicados en el ranquin del coeficiente citado. 
Tabla 4. Aplicación del coeficiente de Gini

\begin{tabular}{|c|c|c|}
\hline País & Coeficiente Gini & Año \\
\hline Ucrania & 0,2500 & 2016 \\
\hline Eslovenia & 0,2540 & 2015 \\
\hline Belarús & 0,2540 & 2017 \\
\hline República Checa & 0,2590 & 2017 \\
\hline República de Moldova & 0,2590 & 2017 \\
\hline República Eslovaca & 0,2650 & 2015 \\
\hline Azerbaiyán & 0,2660 & 2005 \\
\hline Finlandia & 0,2710 & 2015 \\
\hline Kirguistán & 0,2730 & 2017 \\
\hline Noruega & 0,2750 & 2015 \\
\hline Kazajstán & 0,2750 & 2015 \\
\hline Argelia & 0,2760 & 2011 \\
\hline Bélgica & 0,2770 & 2015 \\
\hline Islandia & 0,2780 & 2014 \\
\hline Países Bajos & 0,2820 & 2015 \\
\hline Dinamarca & 0,2820 & 2015 \\
\hline Albania & 0,2900 & 2012 \\
\hline Suecia & 0,2920 & 2015 \\
\hline Malta & 0,2940 & 2015 \\
\hline Iraq & 29,50 & \\
\hline & & 2012 \\
\hline & & \\
\hline
\end{tabular}

Fuente. Elaboración propia con base en datos publicados por Indexmundi, s. f.

En el caso de Latinoamérica los resultados se exponen en la tabla 5, también con diferentes fuentes de origen de datos. Si solo se considera como fuente el Banco Mundial (2016), el país con mejor resultado es Uruguay -0,3950_, luego Argentina - 0,4120-. Perú presenta 0,438. Cuando se aplica este coeficiente, como se cita en el párrafo anterior, se mide la distribución sin diferenciar lo que corresponde a población rural con la urbana. Este dato tiene valor porque no se puede comparar países con alta población en sus ciudades con países con alta población en el campo. Cuando se compara sin tomar en cuenta las diferencias es posible confundir los resultados.

Tabla 5. Coeficiente de Gini en países latinoamericanos

\begin{tabular}{|c|c|c|}
\hline País & Coeficiente Gini & Año \\
\hline Chile & 0,4660 & 2017 \\
\hline Argentina & 0,4120 & 2017 \\
\hline Uruguay & 0,3950 & 2017 \\
\hline Costa Rica & 0,4830 & 2014 \\
\hline Panamá & 0,4990 & 2017 \\
\hline México & 0,4830 & 2016 \\
\hline Venezuela & 0,4690 & 2006 \\
\hline
\end{tabular}




\begin{tabular}{|c|l|l|}
\hline Brasil & 0,5330 & 2017 \\
\hline Ecuador & 0,4470 & 2017 \\
\hline Perú & 0,4330 & 2016 \\
\hline Colombia & 0,4970 & 2017 \\
\hline República Dominicana & 0,4570 & 2016 \\
\hline Paraguay & 0,4880 & 2017 \\
\hline
\end{tabular}

Nota: Chile, Argentina y Uruguay, de acuerdo con el Índice de Desarrollo Humano, se ubican en la categoría «Muy alto». Los once siguientes países latinoamericanos están en la categoría «Alto».

Fuente. Elaboración propia con base en datos publicados por Indexmundi, s. f.

Cuando el país diseña una estrategia y decide establecer una marca puede relacionar los resultados a tres propósitos comerciales principales, tal como lo expresan Lamb, Hair y McDaniel (2006). El primero que permita una clara identificación del producto —bien o servicio - asociándolo al país; el segundo, que los demandantes consideren las empresas del país como un abastecedor seguro en cuanto a la cantidad solicitada y confiable con relación al cumplimiento; y el tercero, que permita la introducción de nuevos productos.

Un país es exitoso cuando sus productos y todo lo que oferta en el mercado internacional tienen una clara ventaja sobre los otros ofertantes. Es así como se puede sacar provecho en el comercio internacional al establecer la marca país y acercarse a sus distintos mercados metas con una oferta distintiva que lo atrae. Considerar una marca país es relacionarse con el posicionamiento país.

El de un producto, una empresa, una comunidad o un país tiene como sustento desarrollar sus actividades con base en la determinación de los objetivos que se tienen, los cuales son parte de un plan. En el caso de un país debe haber un documento que así lo explique. Ahora bien, aunque no se mencione la palabra marketing, se conoce que esa es la intención, como puede ser el caso del Plan Nacional de Competitividad y Productividad (Ministerio de Economía y Finanzas, 2019). Alguna institución del Estado debe hacerlo, pues le corresponde como función que justifica su existencia.

Para tal caso, el proceso de administrar la marca país se denomina branding, a través de lo cual se transmite lo que hace y logra un país mediante acciones que lo distinguen de otros países con los cuales «compite» de alguna manera. Además, muestra a la comunidad mundial qué es como país, para lo que utiliza sus logros en el ámbito social y económico, y explica así por qué deben tener confianza en él y, por tanto, las decisiones que se tomen respecto al país sean lo más favorables posible. Así, por ejemplo, en el comercio ayuda a elegir un producto determinado por que se tiene la confianza de que es un buen producto, sus procesos de producción son los que corresponden e, incluso, superan los estándares. 


\section{Factores que generan la marca país}

$\mathrm{L}$ as actividades que lleva a cabo un país para ser considerado una marca pueden tener como referencia lo que han realizado aquellos países que ya son marca o tienen un posicionamiento reconocido por la comunidad internacional, con la seguridad de que lo que produce tiene los estándares adecuados para el consumo, así como se le reconoce por ser un comprador de insumos y con exigencia en los productos manufacturados con los que se abastece.

La competencia internacional se origina a través de cuánto se ha desarrollado el país a través de su manufactura y el grado de dependencia que tiene en relación de los commodities. Los resultados expuestos de los indicadores exponen cómo los países que tienen una mejor posición son aquellos que tienen una mayor independencia relativa a la exportación de materias primas. En el camino de consolidar una marca, Labarca opina que (comunicación personal, julio de 2019), «en América Latina, me parece que dos ejemplos de éxito corresponden a Perú y Colombia, los que han sabido tener campañas acotadas —en términos conceptuales- que han tenido repercusiones positivas».

La marca se genera por actividades de diferenciación o actividades que son diferentes o únicas respecto a lo que los demás realizan. Se generan como consecuencia de los requerimientos de los mercados objetivo que son los que las define. La marca es un concepto intangible, sin embargo, para que tenga valor debe ser «tangibilizada» con actividades que permitan al consumidor evaluar entre las alternativas que continuamente se le presentan.

Así, la marca permite una visualización favorable del país. El crecimiento económico es importante en el propósito de generar una marca país, sirve como un punto inicial para generarla, toda vez que ahí se generan los recursos económicos y financieros que permiten la sostenibilidad. Tal como lo señala Buitrago (2010, p, 29), cada país funciona competitivamente en un entorno que busca que este se diferencie y se destaque con el papel que desempeña competitivamente de los demás, y aunque existan similitudes culturales, regionales o en las producciones de sus bienes tangibles e intangibles siempre hay algo que hace diferente y único a cada país.

En la exageración, se puede mencionar que los consumidores buscan marca.

Para Chernatony (2008), el uso de las técnicas de marketing, con la finalidad de generar una marca país, es una actividad relativamente nueva, lo cual puede estar influenciado por la creciente competencia comercial entre los países. Estos hacen grandes esfuerzos por perfeccionar el país como una marca, considerando que tal acción les permite cumplir tres grandes objetivos: atraer turistas, atraer inversión extranjera e incrementar el nivel de las exportaciones.

El proceso de construcción de la marca genera un nuevo posicionamiento, el cual es consecuencia de una suma de estrategias que buscan lograr un lugar con más ventaja en la comunidad internacional. Labarca (comunicación personal, julio de 2019) opina que, a fin de obtener un posicionamiento favorable, la condición necesaria es la «generación de una estrategia que sea evaluada y puesta en práctica de forma sistemática. Esto implica que la gestión de la marca país sea una política de Estado y no de gobierno».

Tal como lo expone Buitrago (2010), la construcción de la marca es una labor diaria «que permite mejorar constantemente esos factores de ventajas y fortalezas, disminuir debilidades e innovar para asegurar un éxito en los mercados externos; sin embargo, estas oportunidades requieren de mucho cálculo y responsabilidad». Echeverry et al., por su parte (2013, p, 1123), mencionan que «el concepto de marca país nace de la necesidad de los sectores empresariales y los gobiernos por generar una identidad propia frente a los mercados internacionales». 
Figura 5. Proceso de creación de una marca país

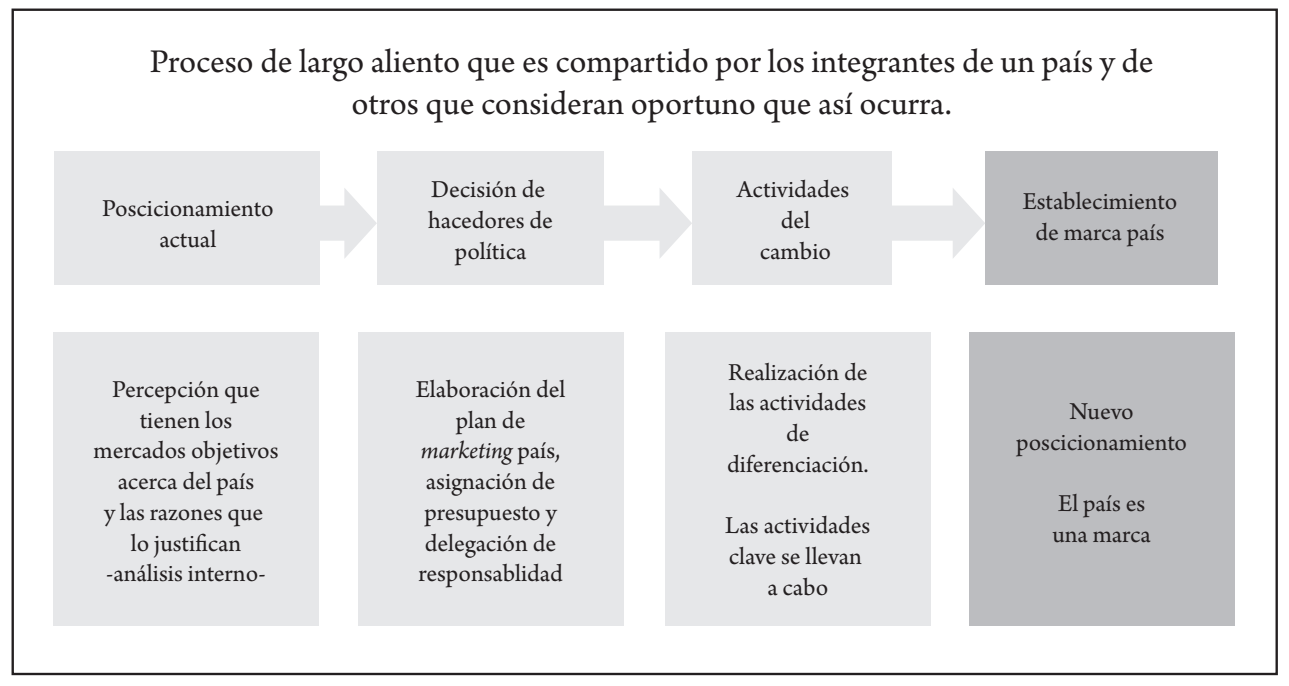

Fuente. Elaboración propia con base en Akatsu, 2008.

Según Akutsu (2008), lo primero que debe realizar un país al querer establecerse como marca es realizar un análisis interno para reconocer las capacidades en los diferentes campos en los que va a actuar (véase la figura 5). Debe continuar con un reconocimiento acerca de lo que hacen los competidores y otros factores del ambiente que pueden afectar. Con este conocimiento los hacedores de política deben diseñar las estrategias específicas en las que se pueden medir los objetivos y los mercados objetivo. Luego de planificar viene la etapa crucial, la que corresponde a la implementación de la estrategia elegida, la cual incluye medidas de evaluación, asignación de recursos humanos, financieros y tecnológicos con capacidad de manejar la estrategia.

De igual forma, el diseño de las estructuras gerenciales y los procesos apropiados, así como las relaciones de dirección a nivel interno y externo. Akatsu (2008) considera que los principios que direccionan la estrategia se basan en dar respuesta a las siguientes tres preguntas - ya sea empresa o país—: ¿dónde estamos ahora?, ¿adónde queremos ir?, y ¿cómo llegamos ahí? Si bien el diseño de la estrategia es un proceso complejo, la respuesta a las tres preguntas proporciona un marco que permite diseñarla.
Cuando un país se establece como marca, la complejidad de las acciones de sostenerla aumenta. Por tal motivo, el país, a través de acciones innovadoras en su proceso de manufactura $y$ desarrollo de intangibles, puede considerar como base los indicadores que se exponen en la figura 4, los cuales pueden ser mediciones relacionadas con la marca país y el uso de las mediciones de riesgo que hacen empresas reconocidas mundialmente, como, por ejemplo, FitchRatings, Standars \& Poors y Moody's Investor Service, instituciones que emiten informes periódicos acerca del riesgo que un país implica para las inversiones, de manera que opinan de las perspectivas de un país en el corto, mediano y largo plazo. Una alternativa para sostener la marca se relaciona con la innovación y está puede ser delegada a las instituciones educativas universitarias y a las instituciones privadas especializadas en investigación. Uno de los beneficios que obtiene un país al establecerse como marca es que le permite relacionar sus productos y empresas como una garantía de que lo comprado o invertido es una decisión correcta que otorga valor a los participantes. El resultado es el siguiente: a) los inversionistas y las empresas locales y extranjeras consideran el país como un agente de 
confianza para mantener relaciones sólidas de largo plazo - es un país que otorga confianza-, y b) la marca facilita las relaciones comerciales, pues se asocia al país de forma positiva a actividades realizadas de forma adecuada.

La idea que expone Anholt (2004) debe considerarse tanto en el origen de los esfuerzos a realizar como en la consecuencia de establecer una marca país. Esto se relaciona con lo que se plantea de ampliar la cartera de productos exportables, pues debe estar claro que una imagen de marca país va a afectar su destino económico, cultural y político. Por tanto, establecer una marca es un logro de largo plazo y así lo deben entender los actores de este proceso.

Como sinónimo de marca país se encuentra la idea de Keegan y Green (2009), quienes explican que en un mundo globalizado uno de los hechos que se observa es lo que denominan como efecto del país de origen, en el que las categorías de productos se asocian con lo positivo o negativo de un país, motivo por el cual esta asociación debe ser cuidadosamente elaborada para, de esta manera, crear una reputación sostenible, toda vez que el resultado de posicionarse es mantenerse en ese lugar a través de la investigación y la innovación.

El hecho de que un país se considere marca global es un asunto dinámico si se considera que los países compiten por el liderazgo mundial o tienen un mejor lugar en la percepción de los compradores, dependiendo del lugar que considere oportuno ocupar en las categorías de la competencia comercial mundial. En este sentido:

Las percepciones sobre países específicos y las actitudes hacia ellos con frecuencia se extienden a los productos y marcas conocidas originarios de esos países. Estas recepciones contribuyen al «efecto del país de origen»; se vuelven parte de la imagen de la marca y contribuyen al valor de esta $[\ldots]$. Las percepciones y las actitudes sobre los orígenes de un producto pueden ser positivas o negativas [comillas añadidas]. (Keegan y Green, 2009, p. 341)

Cabe la reflexión de Chang (2013) acerca de conocer lo que los países, hoy desarrollados, hicieron para llegar al lugar que ocupan en la actualidad: la población tiene un sistema de salud que garantiza su atención oportuna, un nivel de educación que sirve de modelo para otros países y un mejor nivel de ingreso. Por tanto, los diseñadores de política deben considerar tal experiencia.

No debe ser una copia exacta, sino que cada país debe optar por una estrategia que involucra a la industria y los recursos naturales, de acuerdo con sus propias condiciones y posibilidades. Los países que hoy son sinónimo de marca son países que han establecido su posicionamiento con el soporte interno de la población, la cual se identifica con el proceso y el crecimiento industrial como un compromiso de los inversionistas.

\section{Conclusiones}

\footnotetext{
Cenerar una marca país es un proceso complejo $\mathcal{T}_{\text {y beneficioso para el país, considerando que la }}$ competencia es cada vez más intensa entre países, en relación con sus empresas y productos y, por tanto, debe diferenciarse. Como señalan Echeverry, EstayNiculcar, Herrera y Santamaría (2013):
}

la implementación de la marca país implica acciones que involucren a todos los grupos de interés en una ciudad, una región y un país con la finalidad de comprometerlos en el proceso de desarrollo y consolidación de una imagen con una fuerte proyección interna y externa. 
La posición asimétrica en el comercio internacional explica cómo los países que buscan generar una marca país o tener un mejor posicionamiento encuentran barreras de entrada que pueden superarse si reconocen que tienen tal posibilidad, motivo por el cual fortalecen las condiciones internas - económicas y sociales- que los ayuden a ser diferentes frente a los otros actores. La generación de la identidad es lo más importante.

Es todo un desafío la generación de una marca, puesto que va más allá de las actividades promocionales o las campañas publicitarias, lo cual se debe considerar como una de las acciones a realizar. Para tal caso es importante saber administrarla, delegando tal proceso a organizaciones y personas que actúen de manera colegiada para establecerla. Las ideas de Chang (2013) son importantes a fin de saber cómo iniciar el proceso o qué acciones se deben realizar para lograr y establecerse como una marca país. Los países pueden buscar soluciones iniciales si comienzan a preguntarse cómo los países desarrollados, que tienen un posicionamiento internacional, lo lograron.

La preocupación de los países por generar una marca no solo es de un país determinado, como, por ejemplo, Perú, sino que es de varios países que consideran que tal logro es un beneficio en el comercio internacional, en cuanto permite realizar mayores exportaciones. Por tal motivo, al ser esfuerzo de varios, la competencia se intensifica y logra ventaja quien mejores actividades de diferenciación sostenible realice.

Los cambios estructurales que sostienen el desarrollo de la marca país deben ser medibles, a fin de tener conocimiento de cómo se consolida el país. El IDH puede expresar los resultados de una gestión del esfuerzo. También pueden aplicarse a los indicadores de riesgo país que están relacionados con los inversionistas, quienes también son parte del proceso. Se debe reconocer que este proceso va más allá de la actividad comercial y que tiene como soporte el desarrollo de los sistemas educativo, sanitario y judicial, entre otros, elementos que agregan valor a la imagen del país. En fin, de por sí, la generación de marca implica cambios estructurales.

\section{Referencias}

Akutsu, S. L. (2008). The directions and the key elements of branding Japan. En K. Dinnie (Ed.) Nation Branding: concepts, issues, practice. (209-235). Oxford: Elsevier.

Anholt, S. (2004). Nation-brands and the value of provenance. En Destination branding: creating the unique destination proposition (2a ed.). (314). Massachusetts: Elsevier.

Barrientos, P. (2014). El desarrollo de la marca país: base para posicionarse a través de las exportaciones no tradicionales. Revista Finanzas y Política Económica, 6(1), 115-140. DOI: https://doi.org/10.14718/revfinanzpolitecon.v6.n1.2014.26

Bloom Consulting. (2018). Country Brand Ranking-Tourism edition. Madrid: World Economic Forum.

Buitrago, F. (2010). La marca país como estrategia competitiva en el desarrollo del posicionamiento de un país a nivel nacional e internacional (Trabajo de maestría). Universidad Nacional de La Plata. La Plata, Argentina. Recuperado de http://sedici. unlp.edu.ar/handle/10915/3418

Chang, H. (2013). Patada a la escalera: la verdadera historia del libre comercio. Revista Ensayos de Economía, 23(42), 27-57.

Chernatony, L. (2008). Adapting brand theory to the context of nation branding. En K. Dinnie (Ed.) Nation Branding: concepts, issues, practice. (16-31). Oxford: Elsevier.

Echeverry, L.; Estay-Niculcar, Ch.; Herrera, C.; Santamaría, J. (2013). Desarrollo de marca país y turismo: el caso de estudio de México. Revista Estudios y Perspectivas del Turismo, 22(6), 1121-1139.

Fetscherin, M. (2010). The determinants and measurement of a country brand: the country brand strength index. International Marketing Review, 24(4), 466-479. DOI: https://doi. org/10.1108/02651331011058617

Hernández R.; Fernández, C.; Baptista, P. (2014). Metodología de la investigación. México D.F.: McGraw-Hill.

Indexmundi. (s. f.). Índice de Gini. Ranking de países. Recuperado de https://www.indexmundi.com/es/datos/indicadores/ SI.POV.GINI/rankings

Iranzo, S. (2008). Introducción al riesgo país. Madrid: Banco de España.

Kaneva, N. (2011). Nation branding: toward an agenda for critical research. International Journal of Communication, 5, 117-141.

Keegan, W.; Green, M. (2009). Marketing internacional (5 $5^{\mathrm{a}}$ ed.). México: Pearson Educación.

Kotler, P.; Jatusripitak, S. y Maesincee, S. (1998). El marketing de las naciones, una aproximación estratégica a la creación de la riqueza nacional. Buenos Aires: Paidos.

Kotler, P.; Keller, K. (2012). Dirección de marketing (14a ed.). México: Pearson Educación. 
Lamb, Ch.; Hair, J.; McDaniel, C. (2006). Fundamentos de marketing ( $4^{\mathrm{a}}$ ed.). Ciudad de México: International Thomson Editores.

Legatum Institute. (2019). The Legatum Prosperity Index, a tool for tranformation (13a ed.). Londres: Legatum Institute.

Ministerio de Economía y Finanzas. (2002). Determinantes del riesgo país en el Perú: una aproximación. Lima: Ministerio de Economía y Finanzas.

Ministerio de Economía y Finanzas. (2019). Plan Nacional de Competitividad y Productividad 2019-2030. Lima: Ministerio de Economía y Finanzas.

Ohmae, K. (2005). El próximo escenario global: desafío y oportunidades en un mundo sin fronteras. Madrid: McGraw-Hill Interamericana.

Porter, M. (2009). Ser competitivo (6 $6^{\mathrm{a}}$ ed.). Madrid: Deusto.

PNUD (Programa de las Naciones Unidas para el Desarrollo). (2018).Índices e indicadores de desarrollo humano: actualización estadística de 2018. Nueva York: PNUD. 